\title{
Monotherapy versus Combination Therapy for the Treatment of Pseudomonas Aeruginosa in Cystic Fibrosis Patients
}

\author{
Alkhoury $\mathrm{R}^{1}$, Kinkar $\mathrm{E}^{2}$, Nokhbeh $\mathrm{R}^{3}$ and Saleh $\mathrm{MT}^{*^{2}}$ \\ ${ }^{1}$ Medical University of the Americas, Devens, Massachusetts, USA \\ ${ }^{2}$ Department of Biology, Laurentian University, Sudbury, ON, Canada \\ ${ }^{3}$ Health Sciences North Research Institute, Sudbury, ON, Canada
}

*Corresponding author: Saleh MT, Department of Biology, Laurentian University, Sudbury, ON, P3E 2C6, Canada, Fax: 705-675-4859, Tel.: 705-675-1151, E-mail: msaleh@laurentian.ca

Citation: Alkhoury R, Kinkar E, Nokhbeh R, Saleh MT (2018) Monotherapy Versus Combination Therapy for the Treatment of Pseudomonas Aeruginosa in Cystic Fibrosis Patients. J Antibio Res 2(1): 105

Received Date: March 02, 2018 Accepted Date: June 20, 2018 Published Date: June 21, 2018

\begin{abstract}
Background: Infection with Pseudomonas aeruginosa in cystic fibrosis (CF) patients is a strong contributor to respiratory failure and associated mortality. Bacteria colonizing a cystic fibrosis lung commonly form biofilms that greatly contribute to increased antibiotic resistance and hypermutability. Antimicrobial treatment in these cases can be either through the use of a single agent (monotherapy) or through a combination of agents (combination therapy).

Methods: Statistical analysis using the t-test and other tests were applied to clinical in vivo and in vitro studies on the effective of chemotherapeutic treatment of cystic fibrosis. Particular emphasis was on studies published since 2005, not older. The objective was to assess the relative effectiveness of monotherapy versus combination therapy.

Results: Out of 12 studies, it was found that 7/12 studies concluded combination therapy to be more effective than monotherapy, $2 / 12$ studies praised monotherapy over combination therapy, and 3/12 studies showed similar effectiveness between both treatment options. Combination therapy has proven to be superior to monotherapy in overall effectiveness, and resistance development. Additionally, combination therapy has the benefit of potentially reducing the concentrations of the utilized antibiotics, alleviating latent side effects and toxicities associated with long term management. Future studies should focus on comparing novel agents in unison with conventional, inhaled antibiotics.

Conclusion: Analysis of reports on the efficacy of various use of antimicrobials reveal that combination therapy is more effective than monotherapy.
\end{abstract}

Keywords: Pseudomonas Aeruginosa; Combination Therapy; Cystic Fibrosis; Antibiotics; Monotherapy

\section{Introduction}

Cystic Fibrosis (CF) patients exhibit multiple symptoms but mortality mainly stems from debilitating pulmonary infections [1]. Disequilibrium of electrolyte movements and excessive mucus build up contribute to ineffective bronchopulmonary ciliary movements [2]. Lack of protective ciliary propulsion predisposes patients to opportunistic infections that may become fatal if not managed properly. Patients with CF may commonly experience lung infections by Pseudomonas aeruginosa, Staphylococcus aureus and Haemophilus influenzae [1]. However, infections with P. aeruginosa are among the most common cause of mortality in $\mathrm{CF}$ patients and its corresponding treatment will be investigated in this study [3]. P. aeruginosa is a robust, mobile, gram-negative bacterium with an increasing resistance to multiple therapies and a tendency to form chronic infections when located in the pulmonary tract [4-6]. Pathogenicity stems from the bacteria's ability to evade host immune defenses and associated production of a biofilm under the right conditions; such as that of a cystic fibrosis lung [6]. Biofilm production is of particular concern as it contributes to bacterial stability and antibiotic resistance by acting as a protective barrier [7]. Complete eradication of $P$. aeruginosa is proving to be more difficult as treatment-resistant strains seem to be continuously evolving and eluding prescribed therapies [8].

Onset of treatment plays a key role in the survival of infected CF patients. Early diagnosis and treatment strongly impact a patient's prognosis, and reduce potential advancement to a chronic stage. Early therapy can also provide a longer infection-free period where patients can go an average of 18 months between two independent episodes of $P$. aeruginosa infections. Prompt treatment has proven to be cost-efficient through its ability to reduce a patient's hospital stay and decrease long-term hospital visits. In 
addition, $P$. aeruginosa isolates were found to be more susceptible when antibiotic treatment was initiated earlier [9]. Although early treatment is beneficial, prophylactic therapy may not be as transparent. One study has advocated avoiding prophylaxis due to an observed increase in other gram-negative bacterial infections, while another study has demonstrated promise in its use through an observed delay in acquisition and progression of a potential infection [10,11].

The treatment of cystic fibrosis includes a complicated list aimed at alleviating various symptoms the patient may have. Therapy may target potential infections (antibiotics), stabilize an airway (bronchodilators), reduce mucus production (mucolytics), reduce inflammation and pain (anti-inflammatory), replace pancreatic enzymes, and alter genomic mutations (gene therapy) [1,12]. Commonly prescribed antibiotics given to CF patients with a P. aeruginosa infection include colistin, tobramycin, fluoroquinolones and aztreonam [12,13]. Inhalation of the prescribed antibiotics has been chosen as the best route for drug administration [11]. Inhalation deposits the drug directly into the respiratory tract, resulting in increased local concentration and lower systemic concentration; inadvertently decreasing associated drug toxicities due to low systemic penetrance [1,14]. However, disadvantages of inhaled antibiotics exist and their use may be contraindicated in young patients with cystic fibrosis [1]. Although other treatment routes are available (iv, oral), they are often used in combination with an inhaled antibiotic. Currently, there is no universally agreed-upon regimen for $P$. aeruginosa eradication $[1,15]$. In addition, treatment is dependent on the selected antibiotic, route of administration, and whether the respective drug will be given alone, or in conjunction with another antibiotic. The aim of this study was to analyze literature from the past 11 years focussed on the effectiveness of antimicrobial monotherapy, combinations therapy, or both, in CF patients or bacterial isolates from CF patients. Specifically, this study was undertaken to compare the effectiveness of monotherapy versus combination therapy in the management and treatment of CF.

\section{Methods}

\section{Search Strategy}

Search Engines: Pubmed, Google Scholar

Search Terms: "Cystic Fibrosis", "Pseudomonas aeruginosa", "Inhaled”, "Combination", "Colistin”, "Tobramycin”. Terms were used in various ways, either alone or in combination. Some studies were extracted from the references and 'related/similar items' of previously explored articles. A total of 12 articles were chosen to be suitable for this review (Figure 1).

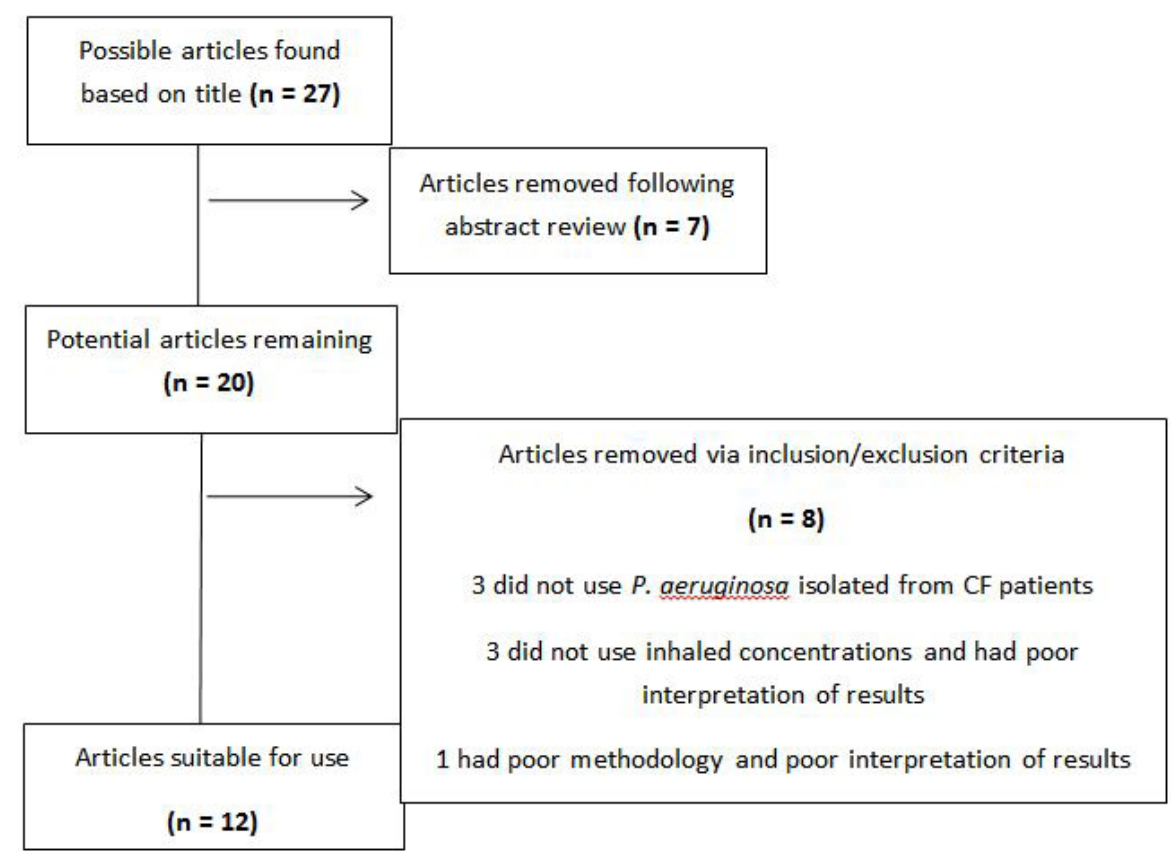

Figure 1: A step-wise breakdown of the accumulated search results. Article were removed through abstract review and implementation of the inclusion/exclusion criteria, resulting in 12 articles suitable for use

\section{Search Criteria}

Inclusions: Must be $\geq$ year 2005; Combination therapy must be compared with monotherapy; cystic fibrosis patients of all ages; in vitro or in vivo studies (human or animal model); in vivo studies should use an inhaled antibiotic for monotherapy, and at least one inhaled antibiotic for combination therapy; in vitro studies must use at least one antibiotic whose concentration is suitable for inhaled human use; biofilm-grown or mucoid $P$. aeruginosa strains that have been directly isolated from cystic fibrosis patients (clinical strains); prospective or retrospective; all geographical locations.

Exclusions: Use of placebo alone as comparison; use of planktonically-grown $P$. aeruginosa alone; use of a laboratory-grown $P$. aeruginosa (wild-type PA01) strain alone; results without statistical analysis and no potential for manual extraction; cystic fibrosis patients with concurrent infections. 
Quality Selection: Hospital or laboratory setting; $\geq 10$ cystic fibrosis patients analyzed (in vivo)

\section{Calculations}

The standard analytical method for this type of analysis is a meta-analysis method. However, the data available in the literature on these treatment regimens is heterogenous and includes in vivo, in vitro, or both methods of treatments. Because of these factors we opted to treat each study separately and confirm statistical significance for each. Two articles, one written by Hill et al. and the other written by Tre-Hardy M had failed to supply sufficient statistical analysis of certain results $[18,19]$. However, provided information was sufficient to calculate a $p$-value using a t-test. The t-test was performed on WPS Office (spreadsheet) 2016, using the function TTEST. Values of the CFU's in Tre-Hardy et al. (17) were analyzed, and values concerning biofilms only of Figure 1 (array 1) and

\begin{tabular}{|c|c|c|c|c|}
\hline Study & $\begin{array}{l}\text { in vivo/in vitro (If in } \\
\text { vitro, are antibiotic } \\
\text { concentrations used } \\
\text { equivalent to inhaled } \\
\text { use?) }\end{array}$ & Antibiotics Used & $\begin{array}{l}\text { Study Population (Study } \\
\text { Design) }\end{array}$ & Measure of Significance \\
\hline Hill et al. [16] & in vitro (yes) & $\begin{array}{l}\text { Amikacin, Tobramycin, Azithromycin, } \\
\text { Cefepime, Ceftazidime and Ticarcillin- } \\
\text { clavulanate, Ciprofloxacin, Cotrimoxa- } \\
\text { zole (TMP-SMX), Meropenem \& Colistin }\end{array}$ & N/A & $\begin{array}{l}\text { Analysis of variance (ANO- } \\
\text { VA) \& Extrapolated t-test }\end{array}$ \\
\hline $\begin{array}{l}\text { Bozkurt-Guzel } \\
\text { et al. [19] }\end{array}$ & in vitro (Yes) & $\begin{array}{l}\text { Colistin, Meropenem, Amikacin, Cef- } \\
\text { tazidime }\end{array}$ & N/A & N/A \\
\hline $\begin{array}{l}\text { Tre-Hardy et } \\
\text { al. [17] }\end{array}$ & in vitro (yes) & Tobramycin \& Clarithromycin & N/A & Extrapolated t-test \\
\hline $\begin{array}{l}\text { Pamp et al. } \\
{[36]}\end{array}$ & in vitro $(\mathrm{No})$ & Colistin, Ciprofloxacin \& Tetracycline & N/A & N/A \\
\hline $\begin{array}{l}\text { Moreau- Mar- } \\
\text { quis et al. [34] }\end{array}$ & in vitro (Yes) & $\begin{array}{l}\text { Tobramycin, Conalbumin, Desferasirox } \\
\text { \& Desferoxamine Mesylate }\end{array}$ & N/A & $P<0.05$ \\
\hline $\begin{array}{l}\text { Macleod et al. } \\
\quad[25]\end{array}$ & $\begin{array}{l}\text { in vitro (yes) \& in vivo } \\
\text { (rats) }\end{array}$ & Tobramyin \& Fosfomycin & Rats (N/A) & $\begin{array}{l}\text { Predefined Interpretations } \\
\text { of CFU changes \& } P<0.05\end{array}$ \\
\hline $\begin{array}{l}\text { Tre-Hardy et } \\
\text { al. }[18]\end{array}$ & in vitro (yes) & Tobramycin \& Clarithromycin & N/A & Extrapolated t-test \\
\hline $\begin{array}{l}\text { Noah et al. } \\
\quad[22]\end{array}$ & in vivo & Tobramycin \& Ceftazidime & $\begin{array}{l}\text { Children (Randomized, } \\
\text { Prospective Study) }\end{array}$ & $\begin{array}{l}\text { 1-tailed Mann-Whitney- } \\
\text { Wilcoxon test }(P<0.05)\end{array}$ \\
\hline $\begin{array}{l}\text { Bergen et al. } \\
\quad[37]\end{array}$ & in vitro $(\mathrm{No})$ & Colistin \& Imipenem & N/A & $\begin{array}{l}\text { Predefined Interpretations } \\
\text { of CFU changes }\end{array}$ \\
\hline $\begin{array}{c}\text { Treggiari et al. } \\
{[23]}\end{array}$ & in vivo & Tobramycin \& Ciprofloxacin & $\begin{array}{l}\text { Children (Randomized, } \\
\text { Double-Blind, Prospective } \\
\text { Study) }\end{array}$ & $\begin{array}{c}\text { Computer Software }(P< \\
0.05)\end{array}$ \\
\hline $\begin{array}{l}\text { Bozkurt-Guzel } \\
\text { et al. [38] }\end{array}$ & in vitro (Yes) & $\begin{array}{l}\text { Tobramycin, Colistin Ciprofloxacin \& } \\
\text { CSA-13 }\end{array}$ & N/A & $\begin{array}{c}\mathrm{X} 2 \text { Test } \\
(P<0.05)\end{array}$ \\
\hline Yu et al. [20] & in vitro (yes) & Tobramycin \& Aztreonam & N/A & $\begin{array}{l}\text { Analysis of variance } \\
\text { (ANOVA) }\end{array}$ \\
\hline $\begin{array}{l}\text { Proesmans et } \\
\quad \text { al. [24] }\end{array}$ & in vivo & Ciprofloxacin, Colistin \& Tobramycin & $\begin{array}{c}\text { Children (Open-label, Ran- } \\
\text { domized, Prospective Study) } \\
\text { N/A }\end{array}$ & $\begin{array}{l}\text { Pearson Chi-square test } \\
\qquad(P<0.05)\end{array}$ \\
\hline $\begin{array}{l}\text { Anderson et al. } \\
\text { [39] }\end{array}$ & in vitro (yes) & Tobramycin \& Fosfomycin & N/A & Student t-test $(P<0.05)$ \\
\hline $\begin{array}{l}\text { Lora-Tamayo } \\
\text { et al. }[40]\end{array}$ & in vitro (No) & Colistin \& Doripenem & $\begin{array}{l}\text { All Ages (Cohort, Rand- } \\
\text { omized Study) }\end{array}$ & $\begin{array}{l}\text { Predefined Interpretations } \\
\text { of CFU changes }\end{array}$ \\
\hline Nick et al. [26] & in vitro (yes) \& in vivo & Tobramycin, Azithromycin \& Aztreonam & N/A & $\begin{array}{l}\text { T-test \& ANOVA \& Chi- } \\
\quad \text { Square }(P<0.05)\end{array}$ \\
\hline $\begin{array}{l}\text { Moreau-Mar- } \\
\text { quis et al. [21] }\end{array}$ & in vitro (Yes) & Tobramycin, Aztreonam \& ALX-109 & N/A & $\begin{array}{l}\text { Analysis of variance } \\
\text { (ANOVA) }\end{array}$ \\
\hline $\begin{array}{l}\text { Furiga et al. } \\
\text { [32] }\end{array}$ & in vitro (Yes) & $\begin{array}{l}\text { Ciprofloxacin, Tobramycin, Ceftazidime, } \\
\text { Colistin \& C11 }\end{array}$ & N/A & Student t-test $(p<0.05)$ \\
\hline de Gier et al. & in vitro (N/A) & Tobramycin, Colistin \& LipoAMP & N/A & N/A \\
\hline $\begin{array}{l}\text { Rojo-Molinero } \\
\text { et al. [27] }\end{array}$ & in vitro (yes) & Aztreonam \& Tobramycin & N/A & Student t-test $(P<0.05)$ \\
\hline
\end{tabular}

Table 1: Studies identified in this review as relevant in the initial search. Articles highlighted in red were excluded through implementation of the inclusion/exclusion criteria $(\mathrm{n}=8)$ 
Table 1 (array 2) in the article written by Hill et al. (16) were also analyzed [16,17]. In a similar fashion, a t-test was measured for a second Tre-Hardy article [18]. Data in Table 1 was utilized, and the (+) and (-) signs were converted to numbers, representative of each treatments logarithmic killing potential. More specifically, (-) became 0, (+) became 1, and (++) became 4 . Lastly, a 2009 article written by Macleod et al. had also undergone a t-test evaluation [19]. The use of Pseudomonas only was assessed for a $p$-value, where fosfomycin/tobramycin combination (array 1) was compared with fosfomycin or tobramycin alone (array 2).

\section{Significance}

All chosen articles must utilize any form of statistical analysis to achieve a $p$-value; $p<0.05$ is considered significant. Articles lacking statistical analysis must provide enough data for manual measurement of a $p$-value.

\section{Results}

The search initially revealed 27 potential papers based upon the title alone. However, only 12 articles are suitable for use after abstract, inclusion, and exclusion filtering. The majority of the articles were performed in vitro for effective testing of various antibiotics in a consistent environment, and overall simplicity. Only five studies attempted in vivo analysis, four of which on humans. An accumulative count of all the antibiotics used can be seen in Figure 2.

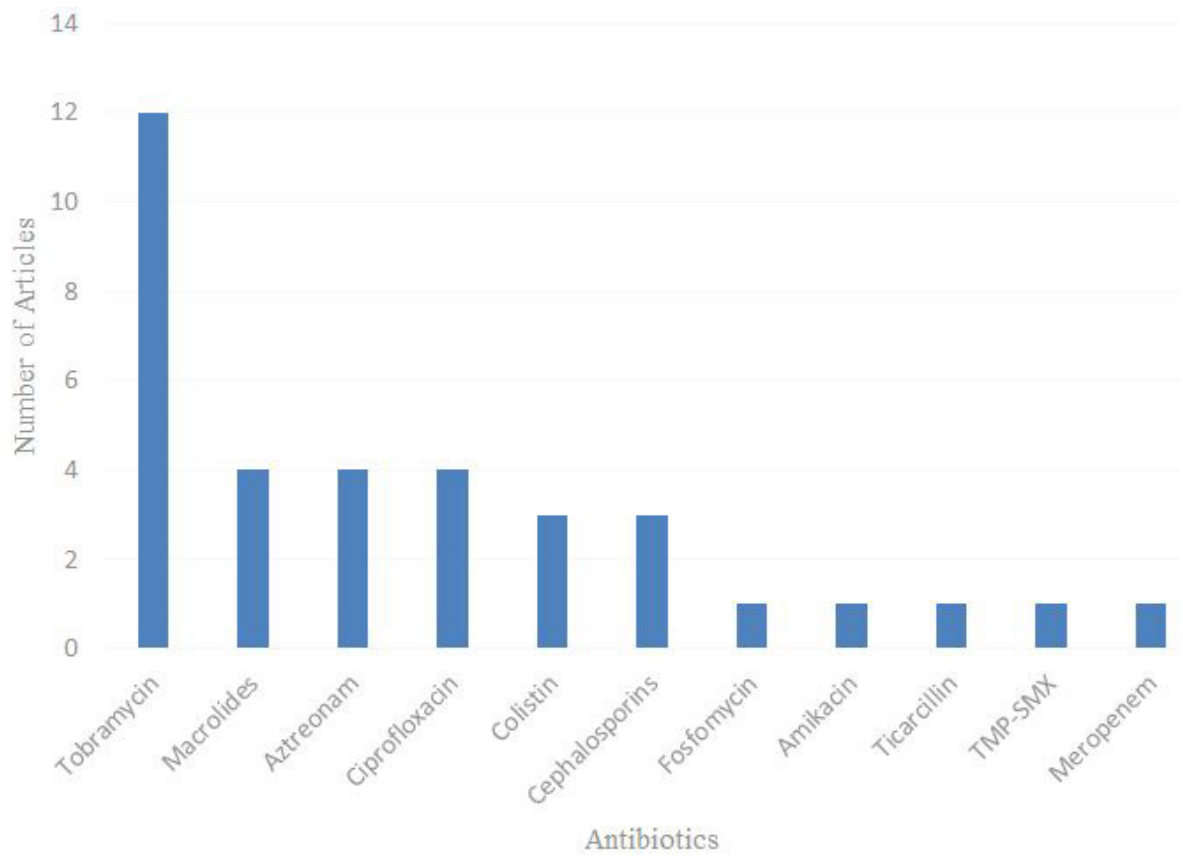

Figure 2: A bar graph representing the number of times a certain antibiotic was used in the extracted articles. As evident in the figure, tobramycin was used in every study

\section{In Vitro Studies}

One the most expansive in vitro studies were published in 2005 by Hill et al. (16) where a total of 10 antibiotics in various combinations were tested [16]. The study analyzed the effectiveness of monotherapy, combination therapy and even triple therapy in $P$. aeruginosa grown in aerobic, anaerobic, and biofilm conditions. Testing individual antibiotics has revealed colistin to be the most effective. A self-measured t-test regarding the activity of single antibiotics and general combination treatments against biofilm-grown bacteria had resulted in an insignificant difference $(\mathrm{p}=0.074)$. However, drug combinations with colistin stood out as a significant bactericidal treatment. Colistin alone was the most effective at killing biofilm-grown P. aeruginosa (49\%), but colistin in combination nearly doubled the bacterial death $(75-81.3 \%)$. The least impressive antibiotics were determined to be amikacin, ciprofloxacin and azithromycin [16]. A 2009 study by Tre-Hardy et al. (17) tested the effectiveness of tobramycin and clarithromycin on various biofilms in different stages of maturity [17]. The study initially tested the antibiotic effect on early biofilm formation and assessed changes through a measurement known as Biofilm Index (BFI); where the lower the value, the higher the concentration of biofilm. BFI measurements at 0,1 , and 24 hours of early biofilm growth revealed that the use of tobramycin and clarithromycin alone or in combination had an insignificant impact on biofilm formation. It is worthy to note that the older biofilm $(24 \mathrm{hr})$ was the least susceptible to any treatment. The study further assessed antibiotic effects on a 24-hour and a 12-day old biofilm preparation. Tobramycin and clarithromycin combination was significantly superior to clarithromycin use alone (24-hr, $\mathrm{p}$ $=0.0197 ; 12$-day, $\mathrm{p}=0.0217)$, but insignificant to tobramycin use alone $(24$-hr, $p=0.425 ; 12$-day, $p=0.460)$. Furthermore, three 12-day old biofilm strains were used in the study to assess the effects of prolonged antibiotic exposure. Although tobramycin was the most effective in the early stages (1-2 days), resistance to both tobramycin and clarithromycin developed quickly and resulted in an increase in bacterial growth. Combination therapy displayed continuous synergistic effects that endured the entire treatment period and resulted in the greatest bacterial decline in $2 / 3$ strains. Combination and tobramycin use had equal killing potential for the final strain [17]. 
Another study only utilized 12-day old biofilms to most accurately resemble the bacterial conditions found inside a cystic fibrosis lung. The researchers obtained $P$. aeruginosa strains from 23 patients with cystic fibrosis [18]. Antibiotic effects were organized in accordance with the resulting logarithmic decrease in colony forming units (CFU). Combination therapy had resulted in a decrease in CFU's/mL greater than $4 \log$ in 11/23 clinical strains. Tobramycin had shown an equal decrease in only $6 / 23$ strains $(p=0.019)$ while clarithromycin was unable to reach such bactericidal levels $(p<<0.05)$. In addition, the study also discovered the presence of different antibiotic susceptibilities and behavior of bacterial isolates from the same patient [18]. A 2011 report by Bozkurt-Guzel et al. introduced a newly synthesized steroid compound with antimicrobial properties [19]. The compound is derived from cholic acid and is part of a related group of compounds called ceragenins. Cationic steroidal CSA-13 was tested alone and in combination with colistin, tobramycin and ciprofloxacin among 50 strains of $P$. aeruginosa. The most effective monotherapy with the lowest $\mathrm{MIC}_{50}$ range was colistin $\left(\mathrm{MIC}_{50}: 0.06-2 \mathrm{mg} / \mathrm{l}\right)$, with CSA-13 a close second $\left(\mathrm{MIC}_{50}: 0.5-4 \mathrm{mg} / \mathrm{l}\right)$. The bactericidal activity of combination therapy was revealed after analysis of different time-kill curve models from two separate bacterial strains. Testing different combinations of antibiotics had revealed the union of colistin and CSA-13 to be the most effective. The duo displayed synergy in $54 \%$ of the strains, significantly greater $(p=0.026)$ than any other combination. Bacterial resistance was found with both tobramycin (6\%) and Ciprofloxacin (24\%) [19].

A study by Yu et al. in 2012 reported on the testing of aztreonam (AZT) and tobramycin (TOB) in the inhibition of biofilm formation (prevention), and in the disruption of preformed biofilm colonies (disruption) [20]. Six clinical P. aeruginosa isolates along with one laboratory (PA01) strain were used for the study, and the results for all are included for completeness. Although the PA01 prevention study showed a significant bacterial decrease with all treatment options (AZT, TOB, AZT/TOB), tobramycin alone had a significantly greater killing ability in comparison to aztreonam alone $(p<0.05)$. Combination therapy and tobramycin alone showed no significant difference. PA01 disruption study resulted in similar findings regarding tobramycin and combination therapy, however, aztreonam alone was found to be ineffective in reducing the CFU of the preformed biofilms. A recent study by Moreau-Marquis et al. also utilized aztreonam and tobramycin, but also studied the use of a novel drug, ALX-109 [21]. ALX-109 is a combination of a bactericidal agent (hypothiocyanite) and an iron-chelating protein (lactoferrin); both elements are naturally occurring components of the innate immune response in CF lungs. As in the previous study, both laboratory and clinical $P$. aeruginosa strains were tested, and the results for both will be included for completeness. Analysis of PA01 biofilm prevention revealed significant effectiveness with use of monotherapy, but bactericidal activity was significantly enhanced once ALX-109 was combined with either antibiotic $(p<0.05)$. Analysis of ALX-109 alone in PA01 biofilm disruption failed to show any activity against the bacterial biofilm defense, yet greatly enhanced the activity of either antibiotic once combined $(p<0.05)[21]$ (Figure 3$)$.

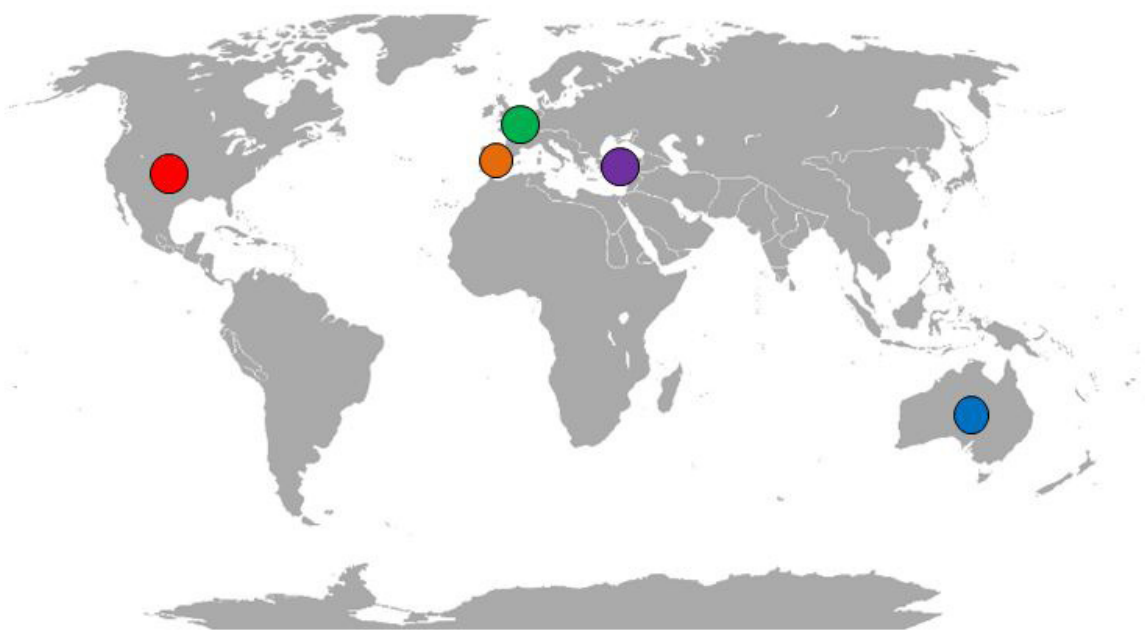

Figure 3: A world map, where coloured dots represent the different locations of the accumulated articles. A total of 6 studies took place in the USA, 3 studies in Belgium, 1 study in Australia, 1 study in Turkey, and 1 study in Spain. The location of a study is dependent upon where the cystic fibrosis patients or sputum was taken from

\section{In Vivo Studies}

A 2010 randomized prospective study by Noah et al. assessed the anti-inflammatory potential of antibiotics through different routes of administration [22]. The study consisted of $15 \mathrm{CF}$ children ( $<16$ years old) who were separated into groups, where $6 / 15$ received inhaled tobramycin only (inhaled group), another 6/15 received intravenous (iv) ceftazidime and inhaled tobramycin (systemic group), and 3/15 received oral ceftazidime and inhaled tobramycin (systemic group). Neutrophils, total cell counts, inflammatory markers (cytokines) and bacterial load were all acquired through careful analysis of bronchoalveolar lavage fluid (BALF). Patients administered systemic antibiotics demonstrated a significant change in neutrophils (PMN/mL) and cell count (total cells $/ \mathrm{mL}$ ). Specifically, combination therapy involving oral ceftazidime and inhaled tobramycin produced the greatest reduction in the percent of inflammatory cells (\% PMNs) and in the total cell count $(p=0.02)$. Although insignificant, complete eradication was achieved in $6 / 7$ patients assigned to the systemic group, and in only $2 / 4$ patient in the inhaled group. 
A unique randomized double-blind trial reported by Treggiari et al. set out to compare cycled therapy (treating patients every 12 weeks) with culture-positive therapy (given to patients when needed) [23]. Every 3 months, the patients may be administered inhaled tobramycin/oral ciprofloxacin or inhaled tobramycin/oral placebo; based upon a cycled schedule or in accordance with a positive $P$. aeruginosa culture. The overall outcome, along with the odds of extracting a positive $P$. aeruginosa culture at a given time $(<20 \%)$ were nearly equal for both cycled and culture-positive administration. The addition of ciprofloxacin was determined to be insignificant $(\mathrm{P}>0.05)$ to the treatment regimen. The inclusion of ciprofloxacin was associated with an increased onset of adverse effects (cough), and bacterial resistance (4\%) than tobramycin. Finally, a 2013 in vivo study by Proesmans et al had focused on comparing inhaled tobramycin (TOB) with the combined use of inhaled colistin and oral ciprofloxacin (CC) [24]. Although 109 children (< 18 years old) were enrolled, 58 had completed the study and were separated equally, such that 29 children were allotted to each treatment group. The primary outcome, $P$. aeruginosa eradication, was achieved in $26 / 29$ patients in the CC group and in 23/29 patients in the TOB group $(p=0.47)$. Time until $P$. aeruginosa reinfection and overall health status following a 2 -year check-up were also documented as secondary outcomes. Patients on CC experienced a median time of 9 months until P. aeruginosa relapse, while TOB patients averaged 5 months between infections $(p=0.608)$. After a 2 year check-up, 14 and 13 children who received $\mathrm{CC}$ and $\mathrm{TOB}$, respectively, were free from infection.

\section{In Vitro \& In Vivo Studies}

MacLeod et al. composed a study that tested the combination of fosfomycin and tobramycin in a multitude of organisms in CF and non-CF patients [25]. Bactericidal effects were determined through MIC50 and MBC (minimal bactericidal concentration) measurements. In vitro assessment of CF-associated P. aeruginosa isolates showed tobramycin use alone to be the most effective agent with a $\mathrm{MIC}_{50}$ of $2 \mathrm{mg} / \mathrm{L}$, combination therapy was a close second with $8 \mathrm{mg} / \mathrm{L}$, and fosfomycin with $64 \mathrm{mg} / \mathrm{L}$. Both tobramycin alone and in combination therapy was $100 \%$ bactericidal against all $P$. aeruginosa isolates, while fosfomycin's bactericidal activity was at $78 \%$. Although synergy was only discovered in a single strain, combination therapy had the longest post-antibiotic effect (PAE), with inhibition lasting up to 3 hours. The in vivo model consisted of $P$. aeruginosa colonization in rat lungs, with intratracheal antibiotic administration 2 times per day for 3 days. Measuring the reduction in CFU's per lung, at various concentrations, had demonstrated significant bacterial reduction with both tobramycin and combination therapy $(p<0.05)$. Fosfomycin however, was unable to produce a significance reduction in the bacterial load at any concentration. In addition, acquired bacterial resistance to each regimen was assessed. A manually performed t-test comparing each treatment regimen has determined insignificant changes in the frequency of acquired resistance over time $(p>0.05)$.

A dual study Nick et al. (26) reported on the interpretation of outcomes concerning 263 CF-patients [26]. This cohort study analyzed unfiltered data that was received from a comparative trial examining the effectiveness of inhaled tobramycin (TOB) and inhaled aztreonam (AZT). Concomitant azithromycin (AZI) use was identified via self-reporting. A total of 128 patients received inhaled tobramycin (71\% reported concomitant azithromycin use), and 135 patients received inhaled aztreonam (67\% reported concomitant azithromycin use). The primary outcome, change in the forced expiratory volume in one second ( $\left.\mathrm{FEV}_{1}\right)$, was assessed in all 4 groups (TOB alone; TOB/AZI; AZT alone; AZT/AZI). The combination of azithromycin and aztreonam produced an insignificant change in the $\mathrm{FEV}_{1}$, when compared with aztreonam use alone. However, in relation to tobramycin use alone, patients taking azithromycin with tobramycin experienced a significant decline in their FEV1 measurements $(p<0.05)$. Through administration of a questionnaire, patients taking TOB/AZI also reported a lower quality of life when compared to any other treatment group. Throughout the examination period, patients taking AZI/TOB combination experienced the greatest need for external intervention due to health issues, requiring emergency antibiotic(s) outside of those used in the study $(p<0.05)$. In continuation, sputum assessments at the end of the study revealed a significant reduction of sputum bacteria $\left(\mathrm{CFU} \log _{10} / \mathrm{g}\right)$ in patients taking TOB alone when compared with combination therapy $(p=0.026)$. An extension study was also performed, where patients taking azithromycin were switched from accompanying tobramycin, to aztreonam use. When TOB/AZI patients were switched to AZT/AZI, a significant improvement was observed concerning the patients FEV1 ( $\mathrm{p}<0.05)$, and the observed level of bacterial reduction became similar to those achieved by the original treatment groups (TOB alone; AZT alone; AZT/AZI).

\section{Discussion}

The objective of this comparative study was to summarize pre-existing data regarding the use of combination treatment in $P$. aeruginosa infected CF patients. Infection with P. aeruginosa is the leading cause of morbidity and mortality in CF patients, and the continuous rise in resistance has created a need for effective, novel treatment methods $[19,26,28,29]$. Only clinical isolates were included in this study because of potential differences that may be unique to bacteria colonizing CF lungs and therefore, utilizing laboratory strains may reduce the potential credibility of an experimental result when implemented on patients. For example, ALX-109 activity differed between laboratory (PA01) and clinical strains with regards to biofilm disruption and Yu et al. Demonstrated aztreonam activity to be nonexistent towards the laboratory strain (PA01) yet produced an effect in all the clinical strains [20,21]. Antimicrobial testing against P. aeruginosa is ideally validated when testing is performed on the pathogen cultured in a biofilm form. Only biofilm growth mimics the innate antibiotic resistance found in $P$. aeruginosa colonizing the airways in a chronic infection $[16,21,27]$. Therefore, the antibiotic treatment that is most effective against biofilm-grown bacteria represents the most suitable clinical option for complete eradication. 
Through a concise sum-up of 12 articles spanning across a 12-year period, the use of the right combination has proven to be more effective in bacterial eradication than use of a single, inhaled antibiotic. In order to precipitate ineffective combinations for future considerations, potential antagonistic behavior was obtained from each study. The addition of azithromycin (AZI) to tobramycin was found to be antagonistic in both studies that had tested it $[16,26]$. These findings are in conflict with a 2016 study that stated the use of oral azithromycin, in combination with a secondary antibiotic, was the treatment of choice for maintenance therapy [28]. Likewise, the addition of ciprofloxacin was insignificant in various trials, and was associated with increased bacterial resistance and adverse effects when combined with tobramycin $[16,19,23,24]$.

Reports of in vivo studies in the literature are limited in numbers and factors including the quality of life, change in lung function, and potential side effects are rarely included in those that are available. Although certain combinations were exceptionally effective, there is insufficient data to extrapolate a single combination as the ultimate tool for clinical use. Lack of consensus may be beneficial since the diffuse utilization of two antibiotics may, in due time, result in an increased incidence of resistant bacteria, and reduce effective eradication in future patients. This review encourages the combined use of one common antibiotic and one novel agent, due to the reduced possibility and frequency of resistance associated with chronic and diffuse utilization.

Concern regarding inhaled antibiotic use lies mainly with the development of bacterial resistance. Although tobramycin was used in every study included in this review, colistin was only utilized in three. With such a low frequency of bacterial resistance to colistin $[16,29]$, upcoming trials are encouraged to include both colistin and tobramycin for effective comparative assessments [16,29].

Anti-inflammatory drugs are projected to reduce inflammation, disease progression, lung damage and rate of bacterial mutation $[22,30]$. Two biofilm inhibitors have demonstrated synergy when combined with conventional antibiotics, including lipidated antimicrobial peptides that occur naturally as part of the host defense (lipoAMPs) and an inhibitor of quorum-sensing involved in regulating biofilm development (C11) [31,32]. Additionally, a peptide extracted from the white blood cells of non-human primates known as rhesus $\theta$-defensin-1 (RTD-1), was found to hold potent antimicrobial activity against CF-isolated $P$. aeruginosa [33]. As seen previously, ALX-109 is a dual agent (bactericidal/iron-binding) that strengthened the effectiveness of the antibiotics it had been paired with [21]. Other iron-chelators of interest include deferoxamine and deferasirox, both proven to strengthen tobramycin when used in combination [34]. Another novel antimicrobial agent is CSA-13, a synthetically made steroidal compound that has proven to be effective with use of various antibiotics in two separate studies $[19,35]$. Future research should test multiple combinations in a single study to determine potential superiority among a certain pair. More in vivo trials should be attempted with longer observational periods for any potential long-term complications. Details concerning the patient's quality of life should be documented, and the researchers should evaluate the most cost-effective approach if possible.

\section{Conclusion}

In a simplified overview, consideration of overall effectiveness, resistance development, and potential toxicities in 12 articles has concluded combination therapy to be the best method of treatment for eradication of Pseudomonas aeruginosa. This review has also shown that a sequential pattern of administration is insignificant to the eradication of $P$. aeruginosa, and when antibiotics are combined, treatment can follow a cycled or culture-positive schedule [25,27]. Future studies should focus on using a traditional antibiotic (colistin or tobramycin) in unison with a novel agent (antibiotic/non-antibiotic) for the most effective, and least likely approach to precipitate resistance with chronic use.

\section{Funding}

The work itself was not funded but the costs associated with publication of the work is funded through the Laurentian University Research Fund.

\section{Author Contributions}

RA performed literature search and analysis and prepared first draft of the manuscript. EK helped in editing the manuscript and in the statistical analysis. RN is a collaborator of the supervisor (MS) and assisted in the study. MS supervised the work, edited the manuscript, and prepared it for submission.

\section{References}

1. Heijerman H, Westerman E, Conway S, Touw D, Doring G, et al. (2009) Inhaled medication and inhalation devices for lung disease in patients with cystic fibrosis: A European consensus. J Cystic Fibrosis 8: 295-315.

2. Elkins RM, Robinson M, Rose RB, Harbour C, Moriarty PC, et al. (2006) A controlled trial of long-term inhaled hypertonic saline in patients with cystic fibrosis. N England J Med 354: 229-40.

3. Govan RWJ, Brown RA, Jones MA (2007) Evolving epidemiology of Pseudomonas aeruginosa and the Burkholderia cepacia complex in cystic fibrosis lung infection. Future Microbiol 2: 153-64.

4. O’Toole AG, Kolter R (1998) Flagellar and twitching motility are necessary for Pseudomonas aeuriginosa biofilm development. Mol Microbiol 30: 295-304.

5. Ly SN, Bulman ZP, Bulitta JB, Baron C, Rao GG, et al. (2016) Optimization of polymyxin B in combination with doripenem to combat mutator pseudomonas aeruginsa. Antimicrob Agents Chemother 60: 2870-80.

6. Murray TS, Egan M, Kazmierczak B (2007) Pseudomonas aeruginosa chronic colonization in cystic fibrosis patients. Current Opin Ped 19: 83-8. 
7. Mah TF, Pitts B, Pellock B, Walker GC, Stewart PS, et al. (2003) A genetic basis for Pseudomonas aeruginosa biofilm antibiotic resistance. Lett Nature 426: 306-10. 8. Jansen G, Mahrt N, Tueffers L, Barbosa C, Harjes M, et al. (2016) Association between clinical antibiotic resistance and susceptibility of Pseudomonas in cystic fibrosis lung. Evol Med Public Health 182-94.

9. Taccetti G, Campana S, Festini F, Mascherini M, Doring G (2005) Early eradication therapy against Pseudomonas aeruginosa in cystic fibrosis patients. European Resp J 26: 458-61.

10. Tramper-Stranders AG, Wolfs TF, Van Haren Noman S, Van Aalderen WM, Nagelkerke AF, et al. (2010) Controlled trial of cycled antibiotic prophylaxis to prevent initial Pseudomonas aeruginosa infection in children with cystic fibrosis. Thorax 65: 915-20.

11. Heinzl B, Eber E, Oberwaldner B, Haas G, Zach SM (2001) Effects of inhaled gentamicin prophylaxis on acquisition of Pseudomonas aeruginosa in children with cystic fibrosis: A pilot study. Ped Pulmonol 33: 32-7.

12. Barry PJ, Jones AM (2015) New and emerging treatments for cystic fibrosis. Drugs 75: 1165-75.

13. Fernandez-Olmos A, Garcia-Castillo M, Maiz L, Baguero F, Canton R (2012) In vitro prevention of Pseudomonas aeruginosa early biofilm formation with antibiotics used in cystic fibrosis patients. Int J Antimicrob Agents 40: 173-6.

14. Ratjen F, Rietschel E, Kasel D, Schwiertz R, Starke K, et al. (2006) Pharmacokinetics of inhaled colistin in patients with cystic fibrosis. J Antimicrob Chemother 57: 306-11.

15. Emiralioglu N, Yalcin E, Meral A, Sener B, Dogru D, et al. (2016) The success of the different eradication therapy regimens for Pseudomonas aeruginosa in cystic fibrosis. J Clin Pharm Ther 41: 419-23.

16. Hill D, Rose B, Pajkos A, Robinson M, Bye P, et al. (2005) Antibiotic susceptibilities of Pseudomonas aeruginosa isolates derived from patients with cystic fibrosis under aerobic, anaerobic, and biofilm conditions. J Clin Microbiol 43: 5085-90.

17. Tre-Hardy M, Mace C, El manssouri N, Vanderbist F, Traore H, et al. (2008) Effects of antibiotic co-administration on young and mature biofilms of cystic fibrosis clinical isolates: the importance of the biofilm model. Int J Antimicrob Agents 33: 40-5.

18. Tre-Hardy M, Traore H, El Manssouri N, Vanderbist F, Vaneechoutte M, et al. (2009) Evaluation of long-term co-administration of tobramycin and clarithromycin in a mature biofilm model of cystic fibrosis clinical isolates of Pseudomoas aeruginosa. Int J Antimicrob Agents 34: 370-4.

19. Bozkurt-Güzel C, Savage BP, Gerceker AA (2011) In vitro activities of the novel ceragenin CSA-13, alone or in combination with colistin, tobramycin, and ciprofloxacin, against Pseudomonas aeruginosa strains isolated from cystic fibrosis patients. Chemotherapy 57: 505-10.

20. Yu Q, Griffin FE, Moreau-Marquis S, Schwartzman DJ, Stanton AB, et al. (2012) In vitro evaluation of tobramycin and aztreonam versus Pseudomonas aeruginosa biofilms on cystic fibrosis-derived human airway epithelial cells. J Antimicrob Chemother 67: 2673-81.

21. Moreau-Marquis S, Coutermarsh B, Stanton AB (2015) Combination of hypothiocyanite and lactoferrin (ALX-109) enhances the ability of tobramycin and aztreonam to eliminate Pseudomonas aeruginosa biofilms growing on cystic fibrosis airway epithelial cells. J Antimicrob Chemother 70: 160-6.

22. Noah TL, Ivins SS, Abode KA, Stewart PW, Michelson PG, et al. (2010) Inhaled versus systemic antibiotics and airways inflammation in children with cystic fibrosis and Pseudomonas. Ped Pulmonol 45: 281-90.

23. Treggiari MM, Retsch-Bogart G, Mayer-Hamblett N, Khan U, Kulich M, et al. (2011) Comparitive efficacy and safety of four randomized regimens to treat early Pseudomonas aeruginosa infection in children with cystic fibrosis. Arch Ped Adol Med 165: 847-56.

24. Proesmans M, Vermeulen F, Boulanger L, Verhaegen J, De Boeck K (2013) Comparison of two treatment regimens for eradication of Pseudomonas aeruginosa infection in children with cystic fibrosis. J Cystic Fibrosis 12: 29-34.

25. MacLeod LD, Barker ML, Sutherland LJ, Moss CS, Gurgel LJ, et al. (2009) Antibacterial activities of a fosfomycin/tobramycin combination: a novel inhaled antibiotic for bronchiectasis. J Antimicrob Chemother 64: 829-36.

26. Nick AJ, Moskowitz MS, Chmiel FJ, Forssen VA, Kim HS (2014) Azithromycin may antagonize inhaled tobramycin when targeting Pseudomonas aeruginosa in cystic fibrosis. Ann Am Thorac Soc 11: 342-50.

27. Rojo-Molinero E, Macia DM, Rubio R, Moya B, Cabot G, et al. (2016) Sequential treatment of biofilms with aztreonam and tobramycin is a novel strategy for combating Pseudomonas aeruginosa chronic respiratory infections. Antimicrob Agents Chemother 60: 2912-22.

28. Talwalkar SJ and Murray ST (2016) The approach to Pseudomonas aeruginosa in cystic fibrosis. Clin Chest Med 37: 69-81.

29. Yayan J, Ghebremedhin B, Rasche K (2015) Antibiotic resistance of Pseudomonas aeruginosa in pneumonia at a single university hospital center in Germany over a 10-year period. PLoS One 10: e0139836.

30. Ciofu O, Riis B, Pressler T, Poulsen EH, Hoiby N (2005) Occurrence of hypermutable Pseudomonas aeruginosa in cystic fibrosis patients is associated with oxidative stress caused by chronic lung inflammation. Antimicrob Agents Chemother 49: 2276-82.

31. de Gier GM, Bauke Albada H, Josten M, Willems R, Leavis H, et al. (2015) Synergistic activity of a short lipidated antimicrobial peptide (lipoAMP) and colistin or tobramycin against Pseudomonas aeruginosa from cystic fibrosis patients. MedChemCommun 7: 148-56.

32. Furiga A, Lajoie B, El Hage S, Baziard G, Roques C (2015) Impairment of Pseudomonas aeruginosa biofilm resistance to antibiotics by combining the drugs with a new quorum-sensing inhibitor. Antimicrob Agents Chemother 60: 1676-86.

33. Beringer MP, Bensman JT, Ho H, Agnello M, Denovel N, Nguyen A (2016) Rhesus $\theta$-defensin-1 (RTD-1) exhibits in vitro and in vivo activity against cystic fibrosis strains of Pseudomonas aeruginosa. J Antimicrob Chemother 71: 181-8.

34. Moreau-Marquis S, O’Toole GA, Stanton BA (2009) Tobramycin and FDA-approved iron chelators eliminate Pseudomonas aeuginosa biofilms on cystic fibrosis cells. Am J Respir Cell Mol Biol 41: 305-13.

35. Chin NJ, Jones NR, Sader SH, Savage BP, Rybak JM (2008) Potential synergy activity of the novel ceragenin, CSA-13, against clinical isolates of Pseudomonas aeruginosa, including multidrug-resistant P. Aeruginosa. J Antimicrob Chemother 61: 365-370.

36. Pamp SJ, Gjermansen M, Johansen, HK, Tolker-Nielsen T (2008) Tolerance to the antimicrobial peptide colistin in Pseudomonas aeruginosa biofilms is linked to metabolically active cells and depends on the pmr and mexAB-oprM genes. Mol Microbiol 68: 223-40.

37. Bergen PJ, Tsuji BT, Bulitta JB, Forrest A, Jacob, et al. (2011) Synergistic killing of multidrug-resistant Pseudomonas aeruginosa at multiple inocula by colistin combined with doripenem in an in vitro pharmacokinetic/pharmacodynamic model. Antimicrob Agents Chemother 55: 5685-95.

38. Bozkurt-Guzel C, Savage PB, Akcali A, Ozbek-Celik B (2014) Potential synergy activity of the novel ceragenin, CSA-13, against carbapenem-resistant Acinetobacter baumannii strains isolated from bacteremia patients. Biomed Res Intl 2014: 710273.

39. Anderson GG, Kenney TF, MacLeod DL, Henig NR, O’toole GA (2013) Eradication of Pseudomonas aeruginosa biofilms on cultured airway cells by a fosfomycin/tobramycin antibiotic combination. Path Dis 67: 39-45. 
40. Lora-Tamayo J, Murillo O, Bergen PJ, Nation RL, Poudyal A, et al. (2014) Activity of colistin combined with doripenem at clinically relevant concentrations against multidrug-resistant Pseudomonas aeruginosa in an in vitro dynamic biofilm model. J Antimicrob Chemother 69: 2434-42. 